\title{
Using 3D Representations of the Nasal Region for Improved Landmarking and Expression Robust Recognition
}

\author{
Jiangning Gao ${ }^{1}$ \\ J.Gao@bath.ac.uk \\ Adrian N Evans ${ }^{1}$ \\ A.N.Evans@bath.ac.uk
}

\author{
${ }^{1}$ Department of Electronic and \\ Electrical Engineering, University \\ of Bath, Bath, UK, BA2 7AY.
}

\begin{abstract}
This paper investigates the performance of different representations of 3D human nasal region for expression robust recognition. By performing evaluations on the depth and surface normal components of the facial surface, the nasal region is shown to be relatively consistent over various expressions, providing motivation for using the nasal region as a biometric. A new efficient landmarking algorithm that thresholds the local surface normal components is proposed and demonstrated to produce an improved recognition performance for nasal curves from both the depth and surface normal components. The use of the Shape Index for feature extraction is also investigated and shown to produce a good recognition performance.
\end{abstract}

\section{Introduction}

Recently proposed 3D face recognition systems address the problem of robustly verifying or identifying human beings in unsupervised environments, especially for variations caused by expression, pose and occlusion, which can destroy the features extracted from captures and significantly affect the intra-class similarity and inter-class dissimilarity. Although using 3D data can facilitate the correction of pose variations and help address the problems caused by occlusions, variations in expressions that lead to muscle movements and deform the face surface still present challenges.

To address this problem many researchers have proposed expression invariant recognition algorithms such as morphable models [1], deformation modelling [2], local shape difference boosting [3, 4], multi-scale and multi-component local normal patterns [5] extended to local binary patterns [6], and sparse representation [7]. A simple and effective alternative to algorithms that use local shape descriptors is to match curves on the facial surface. Drira et al [8] used a set of radial curves emanating from the nose tip to develop a Riemannian framework for analysing shapes of full facial surfaces while Ballihi et al. [9] employed both circular and radial curves to represent the whole facial surface.

Another way to extract expression robust discriminative features is to identify relatively stable structures on the face. Li et al. [5] divided the range image of the whole face into several patches and obtained the weights of each patch. Similarly, Mian et al. [10] explored the recognition performance of different regions of the face and found that both the nasal region and forehead are the most robust structures under varying expressions. However, the forehead is easily occluded by human hair and so presents more challenges 
for feature selection than the nasal region, which has been found to be consistent over natural expressions and occlusions. Chang et al. [11] matched multiple overlapping regions of the 3D nose and its surroundings, obtaining a good recognition performance. Wang et al. [4] also explored differently sized nasal regions by changing the radius of a circle centred on the nose tip, finding that the performance of the nasal region can match that of the whole face while Ballihi et al. [9] found that circular curves around the nasal region produce better recognition performance. All these results show the potential for employing the nasal region to find discriminative features and its significant contribution to face recognition.

Using the stable nasal region with curves matching strategy, Emambakhsh et al. [12] found 75 curves on the nasal region and proposed a new nasal curves matching algorithm for 3D nose recognition which produced good recognition performances on the FRGC [13] and Bosphorus [14] databases. However, in common with many 3D face recognition algorithms, this method focussed on extracting features from the depth information. In addition to depth, the surface normal of each point determines the orientation of the surface and contains information on local shape variations. Therefore, features from the surface normals have significant recognition potential. For example, Zafeiriou et al. obtained the surface normal information from photometric stereo images and used it as a normal face for face recognition [15]. Li et al. used the surface normals calculated from 3D point cloud data, captured using a laser scanner, and demonstrated a good recognition performance [5]. Gao et al. investigated some local patches on the nasal and adjoining cheek region which demonstrated that descriptors found on the surface normals produce better performance than those based on depth [16]. These works provide motivation for the fusion of depth and surface normals to exploit more discriminative features for 3D nose identification.

Inspired by previous work on extracting expression invariant features on the nasal region or the whole face, this paper further investigates the discriminative features for $3 \mathrm{D}$ face recognition. In section 2 , an analysis of the motivation of using nasal region as a biometrics is provided, which shows that the nasal region has great potential to provide discriminative features. For feature extraction, an improved landmark localization algorithm is explained in section 3 and curves found on the nasal region are used to build the feature set. Different types of 3D representation are also explored to extract features, including features extracted from the shape index. The conclusion is given in Section 4.

\section{Analysis of the Motivation of Using Human Nose as a Biometrics}

In this section, three different evaluations are proposed to investigate the benefits of the nasal region, providing the motivation to extract the discriminative features for $3 \mathrm{D}$ nose identification. Calculating the intra-class discrepancy over many kinds of expression demonstrates that the nose region is relatively rigid and its structure is more stable when expression variations occur on the facial surface.

The other two parts concentrate on evaluating the recognition performance using both large and small scale patches on the human face, which also proves that nose region outperforms the other parts and suffers from very few nature occlusions. Both the depth and surface normal maps are considered in the following three evaluations and results presented for all the captures in the Bosphorus database excluding those with occlusions and large pose variations. 


\subsection{Intra-class Dissimilarity under Expressions}

In [17], dissimilarity maps were computed for globally registered faces using the point set features. The maps were built by subtracting the captures with expressions from neutral capture of the same subject. Although these maps provide a good representation of the whole face discrepancy distribution and prove that human nose is relative stable under expressions, only depth information was considered. Li et al. [5] and Gao et al. [16] used three components of surface normal $\left(\mathrm{sn}_{\mathrm{x}}, \mathrm{sn}_{\mathrm{y}}\right.$ and $\left.\mathrm{sn}_{\mathrm{z}}\right)$ calculated from $3 \mathrm{D}$ point clouds and explore the expression invariant discriminative features for recognition, demonstrating the potential of surface normals. Therefore, the three components of surface normals are also considered here. As a preprocessing step, the pose variations are first corrected and all the captures are translated so that they are centred on the nose tip.

The Bosphorus database [14] provides a good choice for expression invariant features extraction. It contains Face Action Units that describe the human face changes when expressions occur on different parts of face: lower, upper and combined. It also contains the basic human expressions of anger, disgust, fear, happy, sadness and surprise. Captures with expression variations are considered in this evaluation and, for illustration, only the combined and basic expressions are shown in Figure 1.

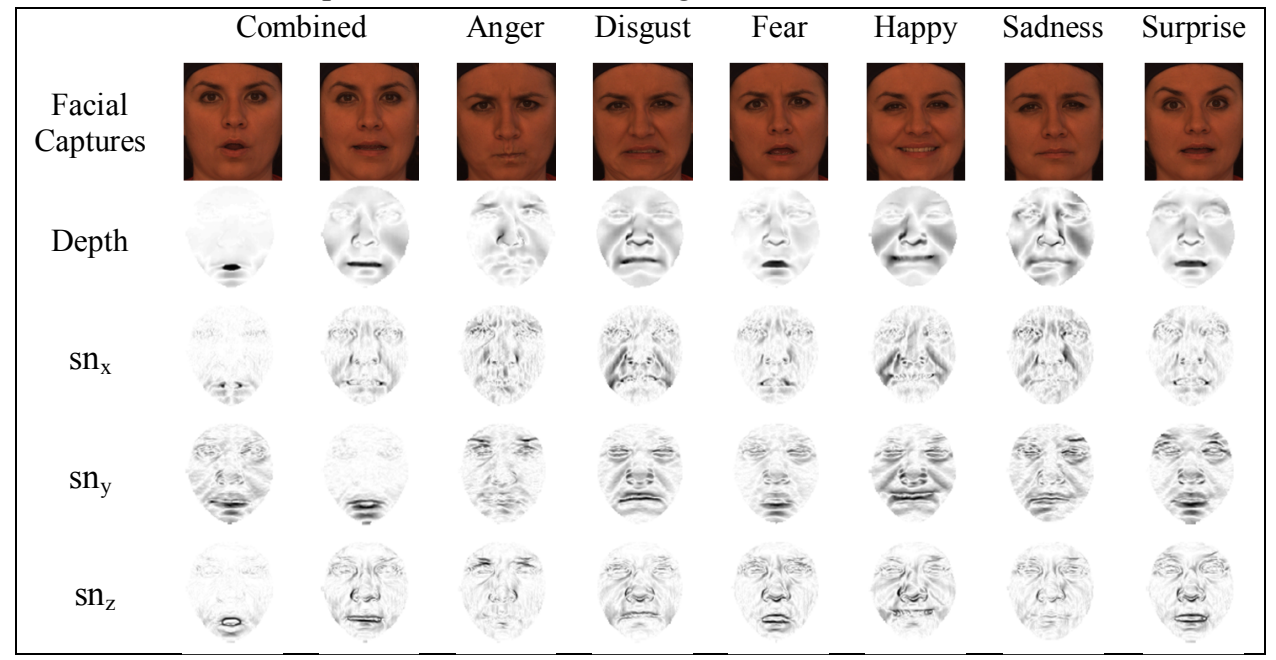

Figure 1: Dissimilarity maps calculated from the captures with expressions and neutral one using 4 components of 3D data on the Bosphorus database. Darker regions show greater dissimilarity on the face.

All the Face Action Units are used for calculation which results in 32 dissimilarity maps of each component (depth, $\mathrm{sn}_{\mathrm{x}}, \mathrm{sn}_{\mathrm{y}}$ and $\mathrm{sn}_{\mathrm{z}}$ ), including 2 combined units, 20 lower units, 4 upper units and 6 basic expressions. To demonstrate the changes on the face, 2 combined units and 6 basic expressions are illustrated in Figure 1. As can be seen from the maps, some small patches on the face show different variance in each component under specific expressions. For example, the cheek bone part is widely regarded as a non-rigid region which suffers more changes under expressions [10]. The depth maps shown in Figure 1 indicate that the cheek bone region has limited stability. However, the surface normals calculated on the cheek are more consistent, which motivates the investigation of different types of discriminative features extracted from the non-rigid regions. Compared 
to other parts on the face, the human nose is the most stable part and also has few natural occlusions.

\subsection{Large Scale Patches Evaluation Using Selected Landmarks}

In previous studies, both $2 \mathrm{D}$ and $3 \mathrm{D}$ facial data are usually divided in to fixed sized patches and their recognition performance evaluated, with Local Binary Patterns (LBP) [6] being the most popular descriptor to extract features on each patch. For example, in [5] all the captures are first resampled to a fixed size and different scales of patches are used for recognition performance evaluation. In addition, the three components of the surface normals are also used to calculate the dissimilarity maps.

However, the main problem of these methods is the difference in the content between patches from different subjects. The underlying reason for this is that human faces possess their own characteristics (e.g. size and curvature) and their structure and distribution is different. Although such discrepancies can preserve the intra-class similarity, it can have a great influence on inter-class dissimilarity. Therefore, in this section, an improved method to correct the discrepancy in content is proposed. In Figure 2(a), from the nose tip eight landmarks are automatically detected: (1) the nose tip, nose root and two alar grooves [12]; (2) two cheek landmarks [16]; (3) middle nose bridge (middle point between nose root and tip) and middle subnasal (symmetrical to middle nose bridge). On the basis of these landmarks, 30 patches are found on the whole face region and each patch is resampled to a fixed size.

A set of LBP values is calculated for each patch and the LBP histogram provides the feature set. The recognition results are shown in Figure 2, where the brighter regions indicate a higher recognition performance. Figure 2(b)-(e) show that, compared to the other parts of the face (cheek, mouth and eye), the nasal region is more discriminative and has more potential to produce a good recognition performance.

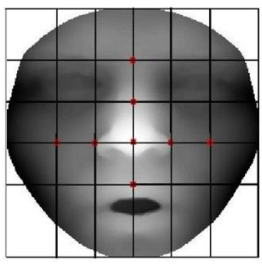

(a) 30 patches

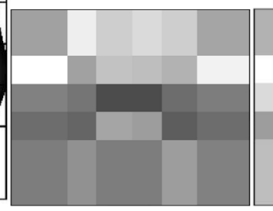

(b) depth

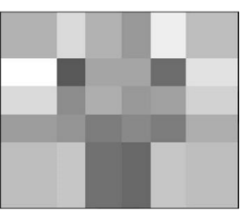

(c) $\mathrm{sn}_{\mathrm{x}}$

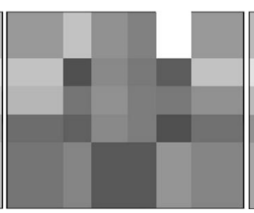

(d) $\operatorname{sn}_{\mathrm{y}}$

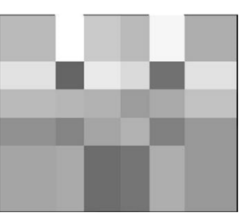

(e) $\mathrm{Sn}_{\mathrm{z}}$

Figure 2: Landmarks based large scale patches and dissimilarity maps of the whole face

\subsection{Local Patches Evaluation}

In addition to the large scale patches for the whole face evaluation, 56 local patches are used to evaluate the discriminatory power on the nasal and surrounding regions. This is an extension work proposed by Gao et al. [16], which divided the middle nasal and adjoining cheek region into 24 patches. To give a comprehensive analysis of the central part of the face another 16 patches including eye and upper nasal region and 16 patches, including the subnasal and upper mouth shown in Figure 3(a), are also considered in this evaluation. As before, the brighter regions indicate a good recognition performance.

The features from each patch are extracted by the local shape descriptor proposed in [16], which is a more effective at finding local features on smaller patches than the original 
Local Binary Pattern. As can be seen from Figure 3(b) to (e), patches from the nasal region generally perform better than the others on the depth, $\mathrm{sn}_{\mathrm{x}}$ and $\mathrm{sn}_{\mathrm{y}}$ maps, especially for the lower nasal part. For the $\mathrm{sn}_{\mathrm{z}}$ map, the nasal region produces a better recognition performance than the eye and upper mouth regions but worse than the adjoining cheek region.

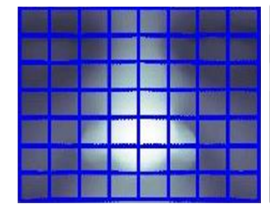

(a) 56 local patches

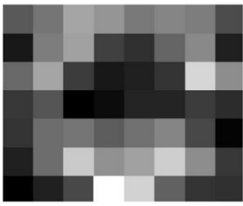

(b) depth

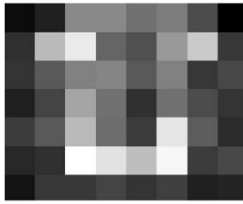

(c) $\mathrm{sn}_{\mathrm{x}}$

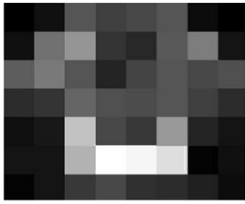

(d) $\mathrm{sn}_{\mathrm{y}}$

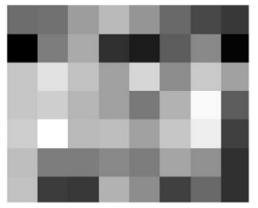

(e) $\mathrm{sn}_{\mathrm{z}}$

Figure 3: The discriminatory power of local patches on and around the nasal region

\section{Landmarks Localization and Feature Extraction}

Landmark localization plays a significant role in region based face recognition algorithms, whose accuracy will directly determine the effectiveness of feature extraction. The intraclass similarity and inter-class dissimilarity may be adversely affected by inconsistent landmarks. In addition, for real-time automatic face recognition system, the landmarking method should also be efficient. Therefore, the aim of the proposed landmarking method is to provide a good compromise of accuracy and efficiency.

On the basis of the method proposed by Emambakhsh et al. [12], a novel landmarking method is proposed by thesholding the three components of surface normals shown in Figure 4(b). Table 1 shows that the intra-class consistency and recognition performance are improved by the proposed method. For feature extraction, the 75 curves shown in Figure 5 are found by connecting selected landmarks in the depth, surface normals and shape index images.

\subsection{Landmarks Localization Using the Surface Normals}

In Figure 4(a), four main landmarks, the nose tip (L9), root (L1) and two alar grooves (L5 and L13), are first detected and the remaining landmarks can be found by dividing the lines (L1 to L5, L5 to L9, L9 to L13 and L13 to L1) into four equivalent parts [2]. Curvature information plays a significant role in $3 \mathrm{D}$ face recognition and can be successfully applied to face detection, image segmentation and landmark localization. Nearly all the region based face recognition algorithms involve curvature. The shape index (SI) can be computed from the principal curvatures, $k_{\max }$ and $k_{\min }$, by

$$
\text { Shape Index }(S I)=\frac{2}{\pi} \arctan \left(\frac{k_{\max }+k_{\min }}{k_{\max }-k_{\min }}\right)
$$

The nose tip is detected by finding the largest convex region on the face, which can be found by thresholding the SI using $-1<$ SI $<-5 / 8$ to produce a binary image $[11,12,18]$. The largest connected component is detected and the centroid is saved as the tip. Nose tip detection is very straightforward and also more robust as the location is modified in each iteration during face alignment.

The nose root and alar grooves are found according to the location of nose tip. First, from the nose tip, a set of planes are defined perpendicular to the $x y$ plane within the 
vertical direction (-15\% to $15 \%)$. This produces a set of curves and the nose tip is located at the maximum of the curves' minima. A similar approach is employed to find nasal alar grooves. However, this method is not always robust for some scenarios: (1) the shape of human nose is diverse and the nasal root is not necessary located at the minimum of each curve, which results in failure detection of some subjects, (2) denoising is still challenging in $3 \mathrm{D}$ face recognition and the algorithm is also sensitive to any residual noise, (3) as it is sensitive to the pose variations, accurate pose alignment is required and (4) occlusions by glasses.

One simple and effective way to address these problems is by adding differential geometry constraints to optimize the location of the candidate landmarks. As a first order surface differential quantity, surface normals demonstrate the orientation of a surface and provide an effective way to localise landmarks. For example, the nasal root detection proposed in [2] can first be used to estimate the location of the candidate root landmarks, C_Root and then the three components of the surface normal can be thresholded using (2). The Root $_{\text {map }}$ shown in the upper part of Figure 4(b), combined with the previous selected region, $C_{-}$Root, produces the final candidate landmarks. A set of $\left(x_{i}, y_{i}\right)$ points indicates the locations of candidate landmarks and the mean value of $x_{i}$ and $y_{i}$ is calculated to find the final nasal root by

$$
\left\{\begin{array}{c}
S n_{x}\left(S n_{x}<T_{x}\right)=\text { positive } ; \\
S n_{y}\left(S n_{y}<T_{y}\right)=\text { positive } \\
S n_{z}\left(S n_{z}>T_{z}\right)=\text { positive } ; \\
\operatorname{Root}_{\text {map }}=S n_{x} \cap S n_{y} \cap S n_{z} \cap C_{-} \text {Root }
\end{array}\right.
$$

Compared to the improvement of nasal root detection, without any prediction only the surface normals are used to localize the nasal alar grooves. The original approximate structure shown in the lower part of Figure 4(b) is obtained by thresholding the $x$ and $z$ components using (3). As the approximation is not connected for some captures, mainly due to residual noise in the data, a morphological closing is then applied. Using the nose tip, the final alar grooves are located at the left and right extrema in horizontal. All the surface normals are normalized before thresholding and the thresholds can be tuned. In this experiment tested on the Bosphorus database, $T_{x}, T_{y}$ and $T_{z}$ of nasal root are set to 0.10 , 0.10 and $0.99 . T_{x}$ and $T_{z}$ of alar grooves are 0.70 and 0.45 .

$$
\left\{\begin{array}{c}
S n_{x}\left(S n_{x}>T_{x}\right)=\text { positive; } \\
S n_{z}\left(S n_{z}<T_{z}\right)=\text { positive; } \\
\text { Alar grooves } \text { map }=S n_{x} \cap S n_{z}
\end{array}\right.
$$

Although the Bosphorus database provides some manually detected landmarks, finding the ground truth for these four landmarks is still very challenging. The effectiveness of these newly detected landmarks can be evaluated by the recognition performance, which will be discussed in the next section. However, the intra-class consistency can be verified by calculating the Euclidean distance between each landmark for captures from the same subject. All the captures are translated to the nose tip and the mean and standard deviation (std) of the distance (in $\mathrm{mm}$ ) of all the subjects are illustrated in Table 1. The proposed landmarking method demonstrates high intra-class consistency in comparison with [12]. 


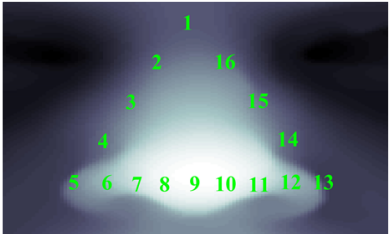

(a) 16 nasal landmarks

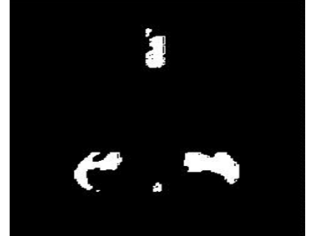

(b) Binary map of selected region

Figure 4: 16 landmarks located on the nasal region and surface normals thesholding

\begin{tabular}{|c|c|c|c|c|}
\hline \multirow{2}{*}{} & \multicolumn{2}{|c|}{ Emambakhsh et al. [12] } & \multicolumn{2}{c|}{ Proposed } \\
\cline { 2 - 5 } & Mean & Std & Mean & Std \\
\hline Root (L1) & 3.7221 & 2.3967 & 3.3789 & 2.3500 \\
\hline Alar_L (L5) & 2.0644 & 1.5028 & 1.9935 & 1.5077 \\
\hline Alar_R (L13) & 2.0619 & 1.5328 & 1.9873 & 1.5357 \\
\hline
\end{tabular}

Table 1: Intra-class landmarks consistency evaluation.

\subsection{Nasal Curves Using Depth, Surface Normals and Shape Index}

In [12], each curve was resampled to 50 points and 28 out of the 75 curves were selected, resulting in a feature set of $28 \times 50$ points from the nasal region. Gao et al. [18] used only 15 points per curve instead of 50 points and proved that fewer points on the nasal and cheek region can also produce an acceptable recognition performance, even with the dimensionality of the feature space greatly reduced. Therefore, 75 nasal curves with 15 points per curve are investigated to extract discriminative features in this paper. To reduce the dimensionality and find the most discriminative curves or curves combination, forward sequential feature selection (FSFS) was applied to the 75 curves, combined with the leaveone-out correlation validation and nearest neighbour classifier.

The rank one recognition rates $\left(R_{1} R R\right)$ obtained from different components of the $3 \mathrm{D}$ face as the size of the feature set increases, as determined by the number of curves selected, are shown in Figure 6. To evaluate the recognition performance of the landmarking, comparisons with the method proposed in [12] (in black), new root only (in blue), new alar grooves only (in green) and both new root and alar grooves (in red) are also shown. Specifically, for all the components $(D, X, Y$ and $Z)$, the recognition performance is improved with the new landmarking algorithms by $\sim 5 \%$ ( $>5 \%$ for $D$ and $X,<5 \%$ for $Y$ and $Z$ ). Although the intra-class consistency of the new nasal tip improves more than alar grooves, the mean and std are still larger, resulting in a lower $\mathrm{R}_{1} \mathrm{RR}$ for the nasal root.

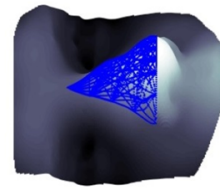

(a) Depth

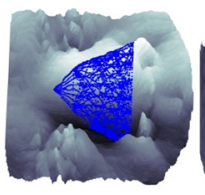

(b) $\mathrm{X}$

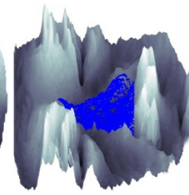

(c) $\mathrm{Y}$

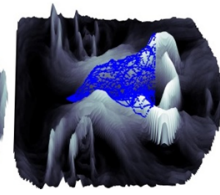

(d) Z

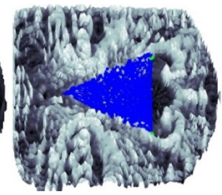

(e) Shape Index

Figure 5: 75 nasal curves representations on five types of data 

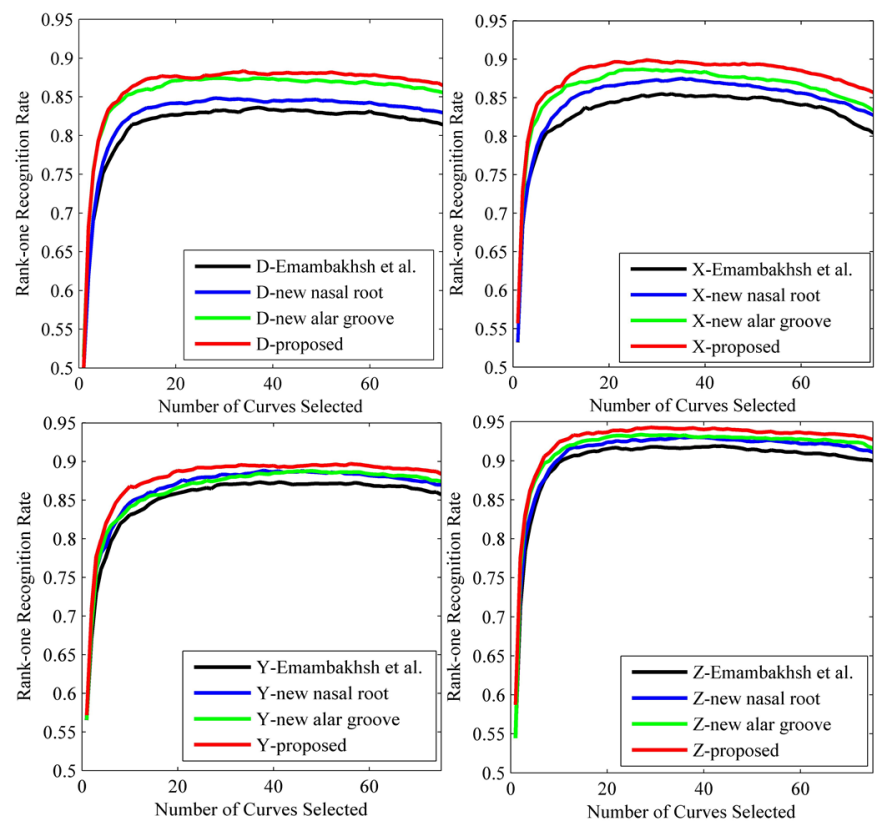

Figure 6: Recognition results by different landmarks on the Bosphorus database.

\subsection{Using the Shape Index for Nose Recognition}

The $2 \mathrm{D}$ representation of the curvature of $3 \mathrm{D}$ surface provided by the $\mathrm{SI}$ is a popular method to characterize the surface patches and many nose, ear and face recognition related algorithms successfully employ it for fiducial feature detection. The SI describes regions of a surface ranging from spherical cup (-1) to spherical cap (+1) with saddle at 0 . A useful property of the SI representation is that curvature is pose invariant.

Although some biometric researchers have used the SI as a new type of discriminative features, it is a seemingly an under explored representation [19]. Huang et al. [20] used a multi-scale local binary pattern depth map together with the SI map to increase the distinctiveness of smooth range faces. Vijayan et al. [21] also explored the usage of SI map for twins 3D face recognition. In [22], a comparison of a series of 3D facial features showed that the SI outperformed PCA and LDA for depth maps while performing worse than the point cloud or surface normal representations.

However, using the SI alone may produce some unexpected problems. The SI coefficients calculated on the planar region are relatively noisy as the curvature of such region is low. Therefore, the SI is not an appropriate representation for describing the whole face region, especially for cheeks. To avoid this drawback, one efficient way is to only SI extract features on less flat regions, for example the nasal region.

Some effective denoising algorithms can be used to smooth the original SI data, for example median filter which is also widely applied in image denoising and depth data smoothing. In addition, curvedness, a positive number that specifies the amount of curvature, proposed by [23], provides a solution to this problem. Therefore, this section focuses on extracting discriminative SI features on the nasal region and evaluating the importance of denoising of the SI calculated from different types of data. 
Figure 7(a) and (b) demonstrate the original SI data from the Bosphorus database calculated by (3). Noisy information can be found in both the frontal and side view, which may cause problems for region segmentation and feature extraction. A recent summary and evaluation of different denoising methods applied to 3D face recognition suggests that the median filter is a good choice for denoising [24]. Therefore, the median filter is applied to the original SI data, resulting in the smoothed data representation shown in Figure 7(c) and (d).

The set of nasal curves used in the previous section are also extracted from the original and denoised SI data, Figure 5(e), and the corresponding recognition performance is shown in Figure 7 (blue curves). The $\mathrm{R}_{1} \mathrm{RR}$ is much higher when the median filter is applied and, in particular, the performance of the curve from the nasal tip to root increases by $\sim 17 \%$ after denoising.

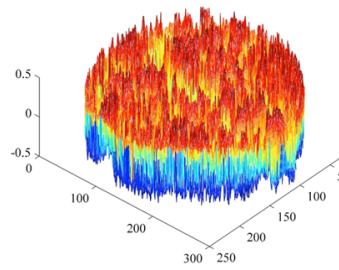

(a) Original SI

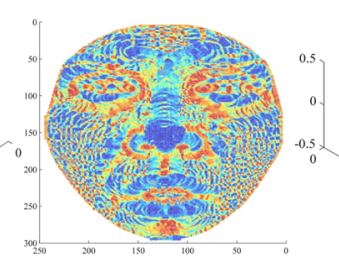

(b) Frontal view

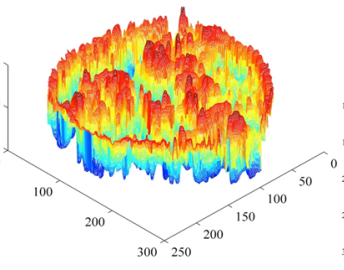

(c) Denoised SI

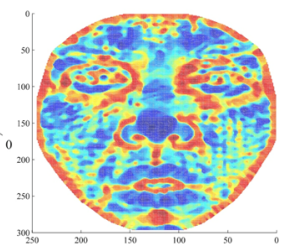

(d) Frontal view (denoised)

Figure 7: Shape Index Denoising on the Bosphorus database

To compare the performance using different types of data, a subset of the FRGC v.2 and Photoface databases [25] are used. In Figure 8, the recognition performance for the Photoface database, shown by black curves, outperforms the Bosphorus (blue) and FRGC v. 2 (red) databases. For the combination of curves, there is $5-10 \% R_{1} R R$ improvement for FRGC and Bosphorus. In contrast, there is no significant increase in the $\mathrm{R}_{1} \mathrm{RR}$ for the curves combination when denoising is applied to the Photoface database, probably because the reconstructed captures of Photoface are relatively smooth and so denoising is of limited benefit.

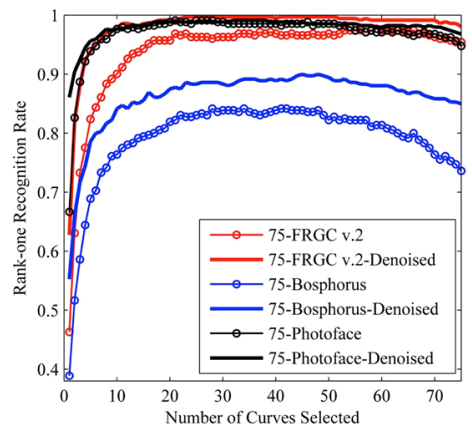

Figure 8: The shape index denoising results tested on three databases.

\section{Conclusions}

In this paper, an investigation of the recognition performance using different representations of 3D nasal region under various expressions is provided. An analysis of the variation of the nasal region over different expression is used to demonstrate that the 
human nose is relatively rigid under expressions and has more potential to extract discriminative features. For feature extraction, there are three main contributions: First, the accuracy of nasal landmarks is improved by thresholding surface normal components. Both the intra-class consistency and recognition performance of the proposed landmarking method are improved. Second, features are extracted from the depth and surface normal components which demonstrates the potential of different 3D representations for providing discriminative features. Finally, using the smoothed SI, after median filtering, is shown to significantly improve the recognition performance.

\section{References}

[1] B. Amberg, R. Knothe, and T. Vetter. Expression invariant 3D face recognition with a Morphable Model. in Proc. 8th IEEE International Conference on Automatic Face \& Gesture Recognition, 2008.

[2] L. Xiaoguang and A. K. Jain. Deformation Modeling for Robust 3D Face Matching. IEEE Transactions on Pattern Analysis and Machine Intelligence, 30(8): 1346-1357, 2008.

[3] W. Yueming, L. Jianzhuang, and T. Xiaoou. Robust 3D Face Recognition by Local Shape Difference Boosting. IEEE Transactions on Pattern Analysis and Machine Intelligence, 32(10):1858-1870, 2010.

[4] W. Yueming, T. Xiaoou, L. Jianzhuang, P. Gang, and X. Rong. 3D Face Recognition by Local Shape Difference Boosting. in Proc. the European Conference on Computer Vision, 2008.

[5] H. Li, D. Huang, J.-M. Morvan, L. Chen, and Y. Wang. Expression-robust 3D face recognition via weighted sparse representation of multi-scale and multicomponent local normal patterns. Neurocomputing, 133(0): 179-193, 2014.

[6] T. Ojala, M. Pietikainen, and T. Maenpaa. Multiresolution gray-scale and rotation invariant texture classification with local binary patterns. IEEE Transactions on Pattern Analysis and Machine Intelligence, 24(7): 971-987, 2002.

[7] L. Xiaoxing, J. Tao, and Z. Hao. Expression-insensitive 3D face recognition using sparse representation. in Proc. IEEE Conference on Computer Vision and Pattern Recognition, 2575-2582, 2009.

[8] H. Drira, B. Ben Amor, A. Srivastava, M. Daoudi, and R. Slama. 3D Face Recognition Under Expressions, Occlusions and Pose Variations. IEEE Transactions on Pattern Analysis and Machine Intelligence, 35(9): 2270 - 2283, 2013.

[9] L. Ballihi, B. Ben Amor, M. Daoudi, A. Srivastava, and D. Aboutajdine. Boosting 3-D-Geometric Features for Efficient Face Recognition and Gender Classification. IEEE Transactions on Information Forensics and Security, 7(6): 1766-1779, 2012.

[10] A. S. Mian, M. Bennamoun, and R. Owens. An Efficient Multimodal 2D-3D Hybrid Approach to Automatic Face Recognition. IEEE Transactions on Pattern Analysis and Machine Intelligence, 29(11): 1927-1943, 2007.

[11] K. I. Chang, W. Bowyer, and P. J. Flynn. Multiple Nose Region Matching for 3D Face Recognition under Varying Facial Expression. IEEE Transactions on Pattern Analysis and Machine Intelligence, 28(10): 1695-1700, 2006.

[12] M. Emambakhsh, A. Evans, and M. Smith. Using nasal curves matching for expression robust 3D nose recognition. in Proc. Biometrics: Theory, Applications and Systems, 2013. 
[13] P. J. Phillips, P. J. Flynn, T. Scruggs, K. W. Bowyer, C. Jin, K. Hoffman, et al.. Overview of the face recognition grand challenge. in Proc. IEEE Computer Society Conference on Computer Vision and Pattern Recognition, 947-954, 2005.

[14] A. Savran, N. Alyüz, H. Dibeklioğlu, O. Çeliktutan, B. Gökberk, B. Sankur, et al.. Bosphorus Database for 3D Face Analysis. in Proc. Biometrics and Identity Management, 47-56, 2008.

[15] S. Zafeiriou, G. A. Atkinson, M. F. Hansen, W. A. P. Smith, V. Argyriou, M. Petrou, et al.. Face Recognition and Verification using Photometric Stereo: The Photoface Database and a Comprehensive Evaluation. IEEE Transactions on Information Forensics and Security, 8(1): 121-135, 2012.

[16] J. Gao and A. N. Evans. Expression robust 3D face recognition by matching multi-component local shape descriptors on the nasal and adjoining cheek regions. in Proc. IEEE International Conference on Automatic Face and Gesture Recogntion, 2015.

[17] N. Alyuz, B. Gokberk, H. Dibeklioglu, and L. Akarun. Component-based registration with curvature descriptors for expression insensitive $3 \mathrm{~d}$ face recognition. in Proc. IEEE International Conference on Automatic Face \& Gesture Recognition, 2008.

[18] J. Gao, M. Emambakhsh, and A. N. Evans. A Low Dimensionality Expression Robust Rejector for 3D Face Recognition. in Proc. International Conference on Pattern Recognition, 506-511, 2014.

[19] M. Hansen. 3D face recognition using photometric stereo. PhD, Faculty of the Environment and Technology, University of the West of England, 2012.

[20] H. Di, Z. Guangpeng, M. Ardabilian, W. Yunhong, and C. Liming. 3D Face recognition using distinctiveness enhanced facial representations and local feature hybrid matching. in Proc. IEEE International Conference on Biometrics: Theory Applications and Systems, 2010.

[21] V. Vijayan, K. W. Bowyer, P. J. Flynn, D. Huang, L. Chen, M. Hansen, et al.. Twins 3D face recognition challenge. in Proc. International Joint Conference on Biometrics, 2011.

[22] B. Gökberk, M. O. İrfanoğlu, and L. Akarun. 3D shape-based face representation and feature extraction for face recognition. Image and Vision Computing, 24: 857869, 2006.

[23] J. J. Koenderink and A. J. van Doorn. Surface shape and curvature scales. Image and Vision Computing, 10(8): 557-564, 1992.

[24] M. Emambakhsh, J. Gao, and A. Evans. An Evaluation of Denoising Algorithms for 3D Face Recognition. in Proc. International Conference on Imaging for Crime Detection and Prevention, 2015.

[25] S. Zafeiriou, M. Hansen, G. Atkinson, V. Argyriou, M. Petrou, M. Smith, et al.. The Photoface database. in Proc. IEEE Computer Society Conference on Computer Vision and Pattern Recognition Workshops, 132-139, 2011. 